\title{
Open-label phase 3 study of diclofenac conjugated to hyaluronate (diclofenac etalhyaluronate: ONO-5704/SI-613) for treatment of osteoarthritis: 1-year follow-up
}

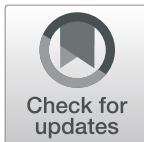

Yoshihiro Nishida ${ }^{1 *}$, Kazuyuki Kano ${ }^{2}$, Taiki Osato ${ }^{2}$ and Takayuki Seo ${ }^{2}$

\begin{abstract}
Background: We evaluated the 1-year safety and efficacy of diclofenac etalhyaluronate (DF-HA), a diclofenacconjugated hyaluronate, in patients with osteoarthritis (OA).

Methods: In this multi-centre, open-label, noncomparative phase 3 study in Japan, patients with a diagnosis of knee, shoulder, elbow, hip, or ankle OA received an intra-articular (IA) injection of DF-HA 30 mg every 4 weeks for 1 year (13 times in total). The safety outcomes included treatment-emergent adverse events (TEAEs) and target joint structural changes by X-ray imaging tests. Efficacy outcomes included joint pain scores on an 11-point numerical rating scale. Concomitant use of analgesics was not restricted.

Results: Overall, 166 eligible patients were enrolled, comprising knee OA $(n=126)$ and other OA $(n=40)$. All TEAEs were experienced by $126 / 166$ patients (75.9\%). The incidence of treatment-related TEAEs was not associated with the treatment period. No significant worsening of joint status was observed in X-ray imaging tests at week 52 or at last assessment. The mean joint pain scores ( \pm standard deviation) were $5.9 \pm 1.2,4.9 \pm 1.9$, and $3.1 \pm 2.3$ at baseline, and weeks 2 and 52, respectively. Improvement of pain score was observed after the first injection and was maintained until week 52 regardless of knee OA or other joint OA.
\end{abstract}

Conclusions: Repeated IA injections of DF-HA every 4 weeks for 1 year were well tolerated with no clinically significant adverse events indicating they might lead to the long-term improvement of OA symptoms. DF-HA might be a useful treatment for patients with OA.

Trial registration number: JapicCTI-183855 (First registered date: 6th February 2018).

Keywords: Osteoarthritis, Diclofenac etalhyaluronate, Phase 3 study, Intra-articular injection

\footnotetext{
* Correspondence: ynishida@med.nagoya-u.ac.jp

'Department of Rehabilitation, Orthopaedic Surgery, Nagoya University Hospital, 65 Tsurumai-cho, Showa-ku, Nagoya, Aichi 466-8560, Japan

Full list of author information is available at the end of the article
}

(c) The Author(s). 2021 Open Access This article is licensed under a Creative Commons Attribution 4.0 International License, which permits use, sharing, adaptation, distribution and reproduction in any medium or format, as long as you give appropriate credit to the original author(s) and the source, provide a link to the Creative Commons licence, and indicate if changes were made. The images or other third party material in this article are included in the article's Creative Commons licence, unless indicated otherwise in a credit line to the material. If material is not included in the article's Creative Commons licence and your intended use is not permitted by statutory regulation or exceeds the permitted use, you will need to obtain permission directly from the copyright holder. To view a copy of this licence, visit http://creativecommons.org/licenses/by/4.0/ The Creative Commons Public Domain Dedication waiver (http://creativecommons.org/publicdomain/zero/1.0/) applies to the data made available in this article, unless otherwise stated in a credit line to the data. 


\section{Background}

Osteoarthritis (OA), the most common joint disorder, occurs frequently in the ageing population [1]. OA primarily affects the knee and hip, but can affect all joints. Major complaints of OA are joint pain, stiffness, and swelling leading to impaired health-related quality of life and activities of daily living [1]. OA can be managed by pharmacological and non-pharmacological therapies regardless of the affected joint. The pharmacological therapy approaches for OA commonly involve the oral or topical administration of nonsteroidal anti-inflammatory drugs (NSAIDs) or selective cyclooxygenase-2 inhibitors as well as intra-articular (IA) corticosteroids or hyaluronan (HA) for knee OA $[2,3]$.

Pharmacological treatment for OA requires a long duration because it is a symptomatic therapy that mainly relieves pain related to OA, which is a chronic disease. Long-term oral administration of NSAIDs increases the risk of gastrointestinal (GI) bleeding [4]. Because of the potential risk related to the long-term use of NSAIDs, it is recommended they are used at the lowest effective dose for the shortest possible time in patients with these risks [2, 3]. There is concern about the long-term use of IA corticosteroids because of the increased risk of accelerated OA progression or adverse joint events [5-7]. Furthermore, IA HA has a favourable safety profile and has been used to treat many patients with OA; however, the efficacy of IA HA for OA remains controversial [2, 3]. Medications available for longterm therapy with sufficient safety and efficacy are anticipated in patients with OA [5-7].

Diclofenac etalhyaluronate (DF-HA: ONO-5704/SI613) is a novel HA derivative consisting of a diclofenac (DF) molecule covalently linked to $\mathrm{HA}$ and was developed as an IA-injectable drug for the treatment of OA [8]. Once DF-HA is injected into the articular cavity of a joint with OA, it gradually releases DF in a sustained manner, which exhibits anti-inflammatory effects lasting up to 28 days, suppression of joint cartilage degeneration and normalization of synovial fluid function $[8,9]$.

The efficacy and safety of IA injections of DF-HA $30 \mathrm{mg}$ were evaluated in patients with knee OA in a randomised placebo-controlled phase 2 study [10] and a confirmatory phase 3 study (unpublished results). In these studies, DF-HA was injected up to six times every 4 weeks during a 6 -month follow-up period, the use of analgesics was limited, and only the knee joint was targeted. Further safety and efficacy evidence for DF-HA is required for its clinical use in OA.

Therefore, we conducted this study (JapicCTI-183,855) to evaluate the safety and efficacy of repeated IA injections of DF-HA $30 \mathrm{mg}$ (every 4 weeks) for 1 year in patients with $\mathrm{OA}$.

\section{Methods}

\section{Study design and patients}

This was a multi-centre, open-label, noncomparative phase 3 study to evaluate the safety (primary objective) and efficacy of multiple doses of DF-HA (Seikagaku Corporation, Tokyo, Japan) injected over 1 year in patients with OA including knee-, shoulder-, elbow-, hip-, or ankle-joint OA in Japan, in accordance with the good clinical practices of the International Conference on Harmonization ( $\mathrm{ICH}$ ) guidelines. A Central Institutional Review Board (C-IRB) approved the study. All investigators are orthopaedic surgeons. Patients provided written informed consent after receiving the consent explanation document approved by the C-IRB. At screening, eligible patients were aged at least 20 years, with a diagnosis of $\mathrm{OA}$ by X-ray imaging regardless of OA grade or stage, with pain for at least 12 weeks, and an 11-point numerical rating scale (NRS) of 4-9 at the target joint. Key exclusion criteria were as follows: invasive procedure to the target joint including arthroscopy within 1 year prior to the start date of screening (except for removal of joint effusion), and radiographically-confirmed serious joint deformation such as osteophyte formation in the target joint which could require surgical intervention during a long-term study.

\section{Intervention}

Screening was performed within 1 week prior to initial injection (week 0). After the screening, eligible patients received IA injection of DF-HA (a prefilled syringe containing DF-HA $30 \mathrm{mg} / 3 \mathrm{~mL}$ ) every 4 weeks for 1 year (13 times in total). To administer DF-HA to the target joint accurately, DF-HA was injected by investigators with experience of IA injection following a manual prepared for the study, which defined the methods and approach to the articular cavity of each target joint. Ultrasound- or X-ray-guided injection was recommended for joints other than the knee joint, which has a large cavity and is usually injected blindly (without imaging assistance or guidance) in normal clinical practice in Japan.

Concomitant use of analgesics was not limited because some patients use DF-HA in combination with analgesics in clinical practice. Injection of other IA products except for $\mathrm{HA}$ at the same target joint was prohibited. However, the use of these medications for the target joint was permitted in cases of treatment for adverse events.

For assessment, patients visited their study centres at weeks $0,2,4$, and every 4 weeks thereafter up to week 52 .

\section{Outcomes}

Safety outcomes were evaluated by treatment-emergent adverse events (TEAEs), clinical laboratory tests, vital signs, target joint examination, and X-ray imaging tests 
to observe structural worsening in the target joint. Causal relationships between TEAEs and DF-HA were judged by the investigators. TEAEs that led to study drug withdrawal were considered significant TEAEs, while those occurring at the injection site or associated with GI disorders, were considered TEAEs of special interest. Similarly, anaphylactic reaction or hypersensitivity were considered TEAEs of special interest because treatment-related anaphylactic reaction occurred in the confirmatory phase 3 study ( $2 / 220$ patients) (unpublished results). Regarding the target joint examination, investigators assessed the joint condition (effusion, swelling, redness, and warmth) on three levels to judge whether the TEAE occurred when the target joint condition had worsened from the screening period. Investigators performed $\mathrm{X}$-ray imaging with reference to the "X-ray imaging procedure manual" prepared separately from the protocol. Each joint X-ray image was captured in the anteroposterior and lateral views. For lower limb joints, $\mathrm{X}$-rays were performed with the patient in the standing position.

Investigators structurally evaluated the worsening of target joint status (osteophyte, joint space narrowing, osteosclerosis, or deformity of epiphysis) by comparing the 24-week and 52-week X-ray images to the baseline images. Evaluation was based on criteria in the "Radiographic Assessment Manual" prepared separately from the protocol for unified evaluation.

Efficacy of DF-HA was assessed by pain scores using the 11-point NRS, patient/physician global assessment by $100-\mathrm{mm}$ visual analogue scale (VAS), quality of life score by Medical Outcomes Study Short-Form 36-Item Health Survey (SF-36), EuroQol 5 dimension (EQ-5D) [11-14], ratio of improved patients, and use of analgesics for the treatment of OA. Joint specific questionnaires were conducted including the Western Ontario and McMaster University Osteoarthritis 3.1 index (WOMAC), Shoulder 36, Patient-Rated Elbow Evaluation of the Japanese Version (PREE-J), and Self-Administered Foot Evaluation Questionnaire (SAFE-Q) for knee/hip, shoulder, elbow, and ankle OA, respectively [15-19]. Because the purpose of this study was the evaluation of the safety of DF-HA, a primary efficacy endpoint was not set.

\section{Statistics}

In accordance with ICH-E1 guidelines, the sample size for knee OA was set at 120 patients to detect TEAEs that occurred with an incidence of $\geq 3 \%$ at $95 \%$ power and considering the study's discontinuation rate. The sample size for the study of OA of the shoulder-, elbow-, hip-, and ankle-joint was set at 40 patients, one-third of the knee OA sample size. Statistical analyses were conducted on knee $\mathrm{OA}$ and other $\mathrm{OA}$ as well as on total OA using SAS version 9.4 (SAS Institute, Cary, NC, USA).
Safety was evaluated in the safety set, which included patients who received treatment at least once. TEAEs were coded using the Medical Dictionary for Regulatory Activities (MedDRA) ver.22.0 and their incidences were calculated. TEAEs were also calculated by the timing of onset. TEAEs associated with GI disorders, anaphylactic reaction, and hypersensitivity were categorized by standardised MedDRA query (broad search). Safety outcomes were summarised in combination with or without systemic NSAIDs such as oral and suppository NSAIDs, and injected amount of HA to assess the safety when DF-HA was used concomitantly with other medications. For the safety evaluation of the amount of IA injected HA concomitantly with DF-HA, subgroup analysis of TEAEs was performed for three groups of patients: those who did not use HA; those who received it concomitantly for $\leq 24$ weeks; and those for $>24$ weeks, where 24 weeks corresponded to half of the planned treatment period of DF-HA for 1 year.

Efficacy was evaluated in the full analysis set (FAS), which included all patients who had at least one evaluation for efficacy after the first injection of DF-HA. Summary statistics of efficacy data were analysed. Patients with pain scores improved by $\geq 30 \%$ from baseline were defined as "responder". For joint pain scores, subgroup analysis was performed in groups stratified according to with or without use of analgesics for OA of the target joint.

\section{Results \\ Patients}

Between February 2018 and August 2019, 172 patients were screened for eligibility from 10 sites and 166 patients were enrolled comprising knee OA $(n=126)$, shoulder OA $(n=15)$, elbow OA $(n=8)$, hip OA $(n=9)$, and ankle OA $(n=8)$ (Fig. 1). All data from 166 enrolled patients were included in the FAS and safety set and 157 patients completed the study (Fig. 1). Most patients received a 3-mL formulation containing $30 \mathrm{mg}$ of DF-HA, but 12 patients did not receive the whole amount of DFHA at least once: knee $(n=7)$, elbow $(n=3)$, and ankle $(n=2)$. The major reasons for dosing volume reduction were injection pain in the joint. For some cases that experienced joint pain, the volume was reduced the next time to prevent pain.

The mean age was 65.9 years and women accounted for $69.9 \%(116 / 166)$ of patients. More women had knee OA compared with other OA. In terms of OA classification, primary OA accounted for $91.6 \%$ (152/166 patients) and secondary OA for $8.4 \%$ (14/166 patients) of patients (Table 1).

\section{Safety}

All TEAEs were experienced by $75.9 \%(126 / 166)$ of patients: $74.6 \%(94 / 126)$ of patients with knee OA and 


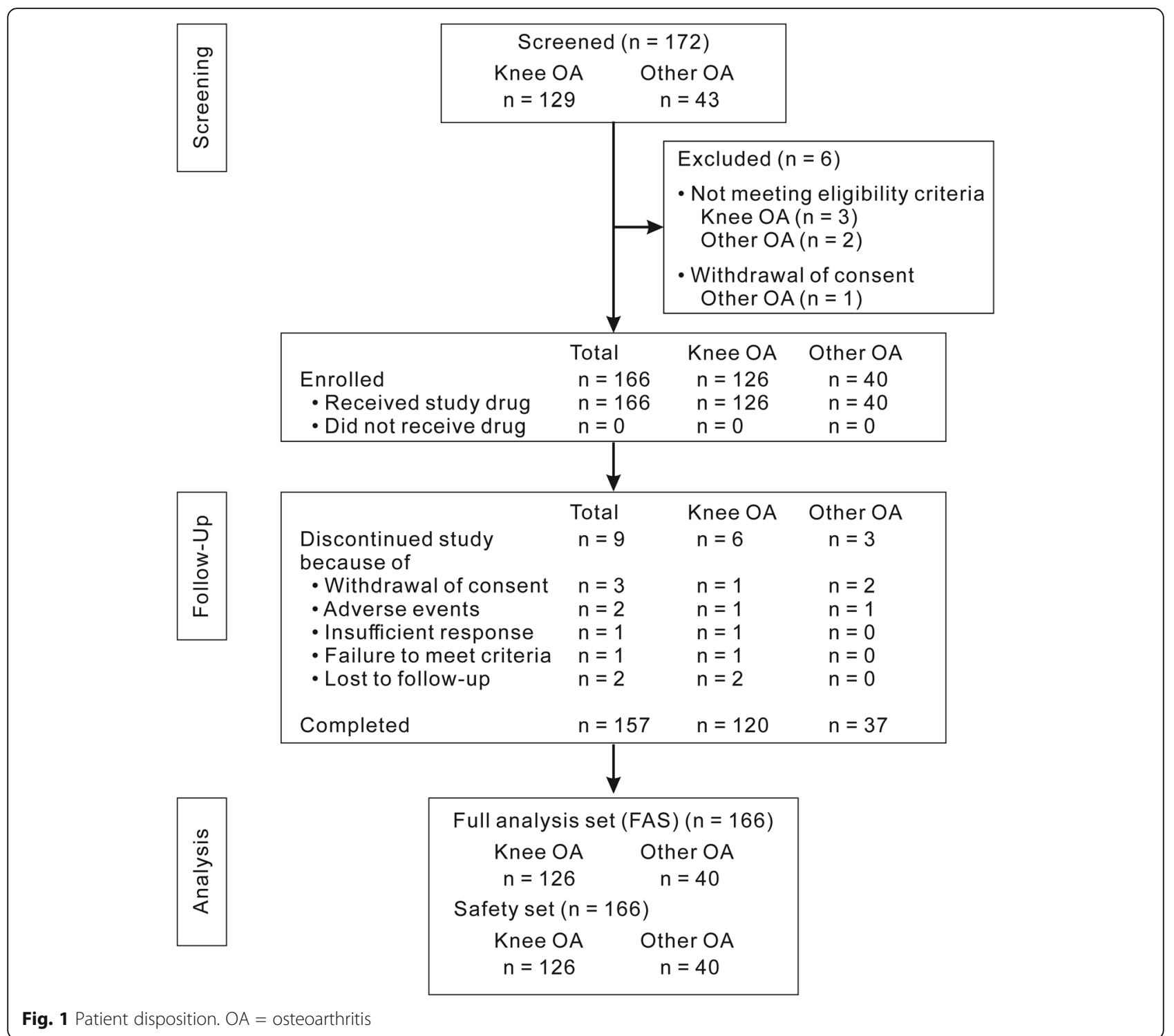

$80.0 \%(32 / 40)$ of patients with other OA. Common TEAEs $(\geq 5 \%)$ were nasopharyngitis, eczema, osteoarthritis, injection site joint pain, and contusion (Table 2). Osteoarthritis was regarded as a TEAE when there was worsening of $\mathrm{OA}$ at the target joint (1/13 of patients) and developing or worsening of OA at non-target joints (12/13 of patients). Treatment-related TEAEs were experienced by $9.0 \%(15 / 166)$ of patients, $7.1 \%(9 / 126)$ of patients with knee OA, and $15.0 \% \quad(6 / 40)$ of patients with other OA. Treatment-related TEAEs occurred in $3.0 \%(5 / 166)$ of patients after the first injection, 3.0\% (5/165) after the second injection, 3.7\% (6/163) after the third injection, 5.0\% (8/160) during the fourth and seventh injections, and 5.7\% (9/158) during the seventh and thirteenth injections. The incidence rate of treatment-related TEAEs was not associated with the treatment period.
No deaths were reported in this study. Twelve serious TEAEs were reported in 10 patients with knee OA. Among them, breast cancer, cerebral infarction, acute myocardial infarction, angina unstable (two events in one patient), and anal polyp were severe. Campylobacter infection, cellulitis (both events in one patient), and appendicitis $(n=2)$ were moderate. Atrial fibrillation and large intestine polyp were mild. Outcomes of all cases were resolved except for breast cancer. The causal relationship to DF-HA was concluded to be unrelated in all cases. One case of treatment-related TEAE associated with palpitations that led to study drug withdrawal was reported in one patient with hip joint OA $(0.6 \%)$ but it was mild and the patient recovered on the same day without treatment (Table 2).

Regarding TEAEs of special interest, treatment-related joint pain at the injection site was reported in $7.8 \%$ of 
Table 1 Demographic and other baseline characteristics in the full analysis set

\begin{tabular}{|c|c|c|c|c|}
\hline Characteristics & & $\begin{array}{l}\text { Knee joint } \\
N=126\end{array}$ & $\begin{array}{l}\text { Other joint } \\
N=40\end{array}$ & $\begin{array}{l}\text { Total } \\
N=166\end{array}$ \\
\hline \multirow[t]{4}{*}{ Age (years), n (\%) } & Mean \pm SD & $67.0 \pm 10.2$ & $62.1 \pm 13.4$ & $65.9 \pm 11.2$ \\
\hline & $<65$ & $51(40.5)$ & $19(47.5)$ & $70(42.2)$ \\
\hline & $\geq 65-75$ & $45(35.7)$ & $14(35.0)$ & $59(35.5)$ \\
\hline & $>75$ & $30(23.8)$ & $7(17.5)$ & $37(22.3)$ \\
\hline \multirow[t]{2}{*}{ Sex, n (\%) } & Male & $33(26.2)$ & $17(42.5)$ & $50(30.1)$ \\
\hline & Female & $93(73.8)$ & $23(57.5)$ & $116(69.9)$ \\
\hline \multirow[t]{3}{*}{ BMI $\left(\mathrm{kg} / \mathrm{m}^{2}\right), \mathrm{n}(\%)$} & Mean \pm SD & $26.06 \pm 4.92$ & $25.33 \pm 4.49$ & $25.88 \pm 4.82$ \\
\hline & $<25.0$ & $54(42.9)$ & $20(50.0)$ & $74(44.6)$ \\
\hline & $\geq 25.0$ & $72(57.1)$ & $20(50.0)$ & $92(55.4)$ \\
\hline \multirow[t]{3}{*}{ Duration of current joint pain (years), n (\%) } & Mean \pm SD & $5.7 \pm 6.4$ & $4.1 \pm 5.3$ & $5.3 \pm 6.2$ \\
\hline & $<1$ & $31(24.6)$ & $13(32.5)$ & $44(26.5)$ \\
\hline & $\geq 1$ & $95(75.4)$ & $27(67.5)$ & $122(73.5)$ \\
\hline \multirow[t]{2}{*}{ Classification of OA, n (\%) } & Primary & $122(96.8)$ & $30(75.0)$ & $152(91.6)$ \\
\hline & Secondary & $4(3.2)$ & $10(25.0)$ & $14(8.4)$ \\
\hline \multirow[t]{5}{*}{ Cause $^{a}, \mathrm{n}(\%)^{\mathrm{b}}$} & Injury & $2(50.0)$ & $1(10.0)$ & $3(21.4)$ \\
\hline & Cuff tear & & $2(20.0)$ & $2(14.3)$ \\
\hline & Meniscus injury & $2(50.0)$ & & $2(14.3)$ \\
\hline & Acetabular dysplasia & & $6(60.0)$ & $6(42.9)$ \\
\hline & Other & 0 & $1(10.0)$ & $1(7.1)$ \\
\hline \multicolumn{5}{|l|}{ Stage of $O A^{c}$} \\
\hline \multirow[t]{4}{*}{ Knee, shoulder and elbow OA, n (\%) } & Grade 1 & $18(14.3)$ & $13(32.5)$ & $31(18.7)$ \\
\hline & Grade 2 & $52(41.3)$ & $5(12.5)$ & $57(34.3)$ \\
\hline & Grade 3 & $43(34.1)$ & $4(10.0)$ & $47(28.3)$ \\
\hline & Grade 4 & $13(10.3)$ & $1(2.5)$ & $14(8.4)$ \\
\hline \multirow[t]{3}{*}{ Hip OA, n (\%) } & Early stage & & $5(12.5)$ & $5(3.0)$ \\
\hline & Advanced stage & & $3(7.5)$ & $3(1.8)$ \\
\hline & End stage & & $1(2.5)$ & $1(0.6)$ \\
\hline \multirow[t]{4}{*}{ Ankle OA, n (\%) } & Stage 1 & & $3(7.5)$ & $3(1.8)$ \\
\hline & Stage 2 & & $1(2.5)$ & $1(0.6)$ \\
\hline & Stage 3 & & $3(7.5)$ & $3(1.8)$ \\
\hline & Stage 4 & & $1(2.5)$ & $1(0.6)$ \\
\hline \multirow[t]{4}{*}{ Joint pain score ${ }^{d}, \mathrm{n}(\%)$} & Mean \pm SD & $6.0 \pm 1.1$ & $5.7 \pm 1.3$ & $5.9 \pm 1.2$ \\
\hline & $<5$ & $15(11.9)$ & $12(30.0)$ & $27(16.3)$ \\
\hline & $\geq 5-7$ & $71(56.3)$ & $19(47.5)$ & $90(54.2)$ \\
\hline & $\geq 7$ & $40(31.7)$ & $9(22.5)$ & $49(29.5)$ \\
\hline
\end{tabular}

$S D$ standard deviation, $B M I$ body mass index, $O A$ osteoarthritis, $K-L$ Kellgren and Lawrence

${ }^{a}$ This assessment was conducted for patients with secondary OA

${ }^{b}$ Percentages are based on the number of patients with secondary OA

In knee, shoulder, and elbow joints, Stage of OA is indicated by K-L grading score. In hip and ankle joints, Stage of OA is indicated by stage of each joint

${ }^{d}$ For the 0 to 10 numerical rating scale for pain intensity, 0 indicates no pain and 10 indicates worst pain

patients. TEAEs associated with GI disorders (gastric ulcer or gastritis erosive), hypersensitivity (e.g., eczema and stomatitis), and anaphylactic reaction (e.g., erythema and urticaria) were observed, but all TEAEs were judged to have no causal relationship to DF-HA (Table 2). For these events of special interest, no serious TEAEs and TEAEs leading to study drug withdrawal or clinically significant events were observed.

For the subgroup analysis of concomitant medication use, the incidence of TEAEs was $63.9 \%$ (46/72) in 
Table 2 Treatment-emergent adverse events in the safety set

\begin{tabular}{|c|c|c|c|c|c|c|}
\hline & \multicolumn{3}{|l|}{ TEAEs } & \multicolumn{3}{|c|}{ Treatment-related TEAEs } \\
\hline & Knee joint & Other joint & Total & Knee joint & Other joint & Total \\
\hline & $N=126$ & $N=40$ & $N=166$ & $N=126$ & $N=40$ & $N=166$ \\
\hline Any events & $94(74.6)$ & $32(80.0)$ & $126(75.9)$ & $9(7.1)$ & $6(15.0)$ & $15(9.0)$ \\
\hline \multicolumn{7}{|l|}{ Common events ( $\geq 5 \%$ ) } \\
\hline Nasopharyngitis & $30(23.8)$ & $11(27.5)$ & $41(24.7)$ & 0 & 0 & 0 \\
\hline Eczema & $6(4.8)$ & $4(10)$ & $10(6.0)$ & 0 & 0 & 0 \\
\hline Osteoarthritis & $11(8.7)$ & $2(5.0)$ & $13(7.8)$ & 0 & 0 & 0 \\
\hline Injection site joint pain & $9(7.1)$ & $5(12.5)$ & $14(8.4)$ & $8(6.3)$ & $5(12.5)$ & $13(7.8)$ \\
\hline Contusion & $13(10.3)$ & $4(10.0)$ & $17(10.2)$ & 0 & 0 & 0 \\
\hline Serious TEAEs & $10(7.9)$ & 0 & $10(6.0)$ & 0 & 0 & 0 \\
\hline Significant TEAEs ${ }^{\mathrm{a}}$ & 0 & $1(2.5)$ & $1(0.6)$ & 0 & $1(2.5)$ & $1(0.6)$ \\
\hline \multicolumn{7}{|l|}{ Special interest events } \\
\hline Events at injection site & $12(9.5)$ & $6(15.0)$ & $18(10.8)$ & $8(6.3)$ & $5(12.5)$ & $13(7.8)$ \\
\hline Gastrointestinal disorders & $2(1.6)$ & 0 & $2(1.2)$ & 0 & 0 & 0 \\
\hline Anaphylactic reaction & $4(3.2)$ & $3(7.5)$ & $7(4.2)$ & 0 & 0 & 0 \\
\hline Hypersensitivity & $16(12.7)$ & $7(17.5)$ & $23(13.9)$ & 0 & 0 & 0 \\
\hline
\end{tabular}

TEAEs were coded using the Medical Dictionary for Regulatory Activities (MedDRA) ver.22.0. OA = osteoarthritis; knee joint = patients with knee OA; Other joint = patients with OA at joints other than knee; NSAIDs = Nonsteroidal anti-inflammatory drugs; total = whole patients treated concomitantly with or without systemic NSAIDs

${ }^{a}$ Treatment-emergent adverse events (TEAEs) leading to study drug withdrawal are defined as significant TEAEs

patients who did not receive NSAIDs compared with $85.1 \%(80 / 94)$ in patients treated concomitantly with NSAIDs, in whom system organ classes "infections and infestations", "musculoskeletal and connective tissue disorders", and "injury, poisoning and procedural complications" were commonly reported. Incidences of GI disorders were $1.4 \%(1 / 72)$ and $1.1 \%(1 / 94)$ in patients treated concomitantly without and with NSAIDs, respectively. Similarly, TEAEs including events at the injection site were not increased in patients receiving IA injection of HA concomitantly with DF-HA over 24 weeks compared with those who received treatment for 24 weeks or less (Table 3 ).

For target joint examination, percentages of patients that had worsened joint effusion, swelling, redness, or warmth compared with baseline were in the ranges of
$1.3-4.2 \%, 1.3-3.8 \%, 0$, and $0 \%-0.6 \%$, respectively, and percentages were not associated with injection times. All events assessed as worsened were judged to not be TEAEs. Regarding X-ray imaging, fractions of patients with structural worsening were $0.6 \%(1 / 166), 2.4 \%$ (4/ $166), 1.8 \%(3 / 166)$, and $0 \%(0 / 166)$ for osteophytes, joint space narrowing, osteosclerosis, and deformity of epiphysis at week 52 or last assessment, respectively. One finding of narrow joint space in a patient with knee OA was judged to be TEAE associated with OA at week 24. A causal relationship to treatment in this case was determined to be unrelated and DF-HA was injected through to week 52, when further changes were not found. All other findings were judged not to be TEAEs. There were no noteworthy changes from the baseline laboratory values and vital signs.

Table 3 Subgroup analysis of TEAEs by concomitant medication

\begin{tabular}{lllll}
\hline & N & TEAEs & Treatment-related TEAEs & Gastrointestinal disorders $^{\mathbf{a}}$ \\
\hline Without NSAIDs & 72 & $46(63.9 \%)$ & $5(6.9 \%)$ & $1(1.4 \%)$ \\
With NSAIDs & 94 & $80(85.1 \%)$ & $10(10.6 \%)$ & $1(1.1 \%)$ \\
& $\mathrm{N}$ & TEAEs & Treatment-related TEAEs & Events at injection site \\
Without HA & 112 & $83(74.1 \%)$ & $12(10.7 \%)$ & $14(12.5 \%)$ \\
With HA for $\leq 24$ weeks & 30 & $23(76.7 \%)$ & $2(6.7 \%)$ & $1(3.3 \%)$ \\
With HA for $>24$ weeks & 24 & $20(83.3 \%)$ & $1(4.2 \%)$ & $3(12.5 \%)$
\end{tabular}

TEAEs treatment-emergent adverse events, HA hyaluronan, NSAIDs nonsteroidal anti-inflammatory drugs

${ }^{\mathrm{a}} \mathrm{Standardised}$ MedDRA Queries (Broad Scope) 


\section{Efficacy}

The mean joint pain scores ( \pm standard deviation) were $5.9 \pm 1.2,4.9 \pm 1.9$, and $3.1 \pm 2.3$ at baseline and at weeks 2 and 52, respectively. Improvement of pain score was observed after the first injection and was maintained until week 52 (Fig. 2). Additionally, similar improvements were noted for the physician global assessment, SF-36 Physical Component Summary, and EQ-5D scores. The proportion of improved patients after each injection was $68.8 \%(108 / 157)$ at week 52 (Table 4).

The time course of pain scores showed an improvement that was similar between knee OA and other joint OA (Fig. 2). WOMAC scores as joint specific questionnaires for the knee joints including subscores to assess stiffness and physical function as well as pain showed continuous improvement in all subscores by repeated injection of DF-HA up to week 52 (Table 5). The score of other joints also showed an improved trend using joint specific questionnaires, although a large variance in values was observed.

Regarding the subgroups treated concomitantly with or without analgesics, the main analgesics for the treatment of OA were NSAIDs and the numbers of patients who used topical and systemic NSAIDs at least once were $111(66.9 \%)$ and $72(43.4 \%)$ patients, respectively. The mean joint pain scores in subgroups treated concomitantly with or without analgesics decreased from $5.9 \pm 1.2$ and $5.7 \pm 1.2$ at baseline to $4.9 \pm 1.9$ and $4.7 \pm 2.2$ at week 2 , and then decreased continuously to $3.2 \pm 2.3$ and $2.4 \pm$
2.4 at week 52, respectively. Thus, there was no difference in the improvement of joint pain scores in both subgroups with or without analgesics.

\section{Discussion}

We evaluated the safety and efficacy of DF-HA repeated injection every 4 weeks for 1 year in patients with OA. For the safety evaluation, injected joints were evaluated in addition to TEAEs by target joint examination and structural changes because NSAIDs were reported to be deleterious for cartilage [20], and the progression of joint destruction, joint space narrowing, or osteonecrosis was reported in OA patients treated with anti-nerve growth factor antibody or continuous treatment of IA corticosteroids $[21,22]$. The incidence of TEAEs did not increase with the repeated injection of DF-HA for 1 year, the pattern and frequency of TEAEs and joint dysfunction were similar to a previous phase 2 study [10] and confirmatory phase 3 study (unpublished results) as well as other studies of IA treatments in OA patients [23]. Regarding TEAE-related anaphylactic reactions and hypersensitivity, significant adverse events such as those observed in the confirmatory phase 3 study were not observed. Of note, clinically significant TEAEs were not reported. For efficacy, an improvement of OA symptoms was observed after the first injection, and this was maintained up to week 52 . Results of a confirmatory phase 3 study indicated that IA injection of DF-HA demonstrated a significant improvement of OA symptoms compared with placebo,

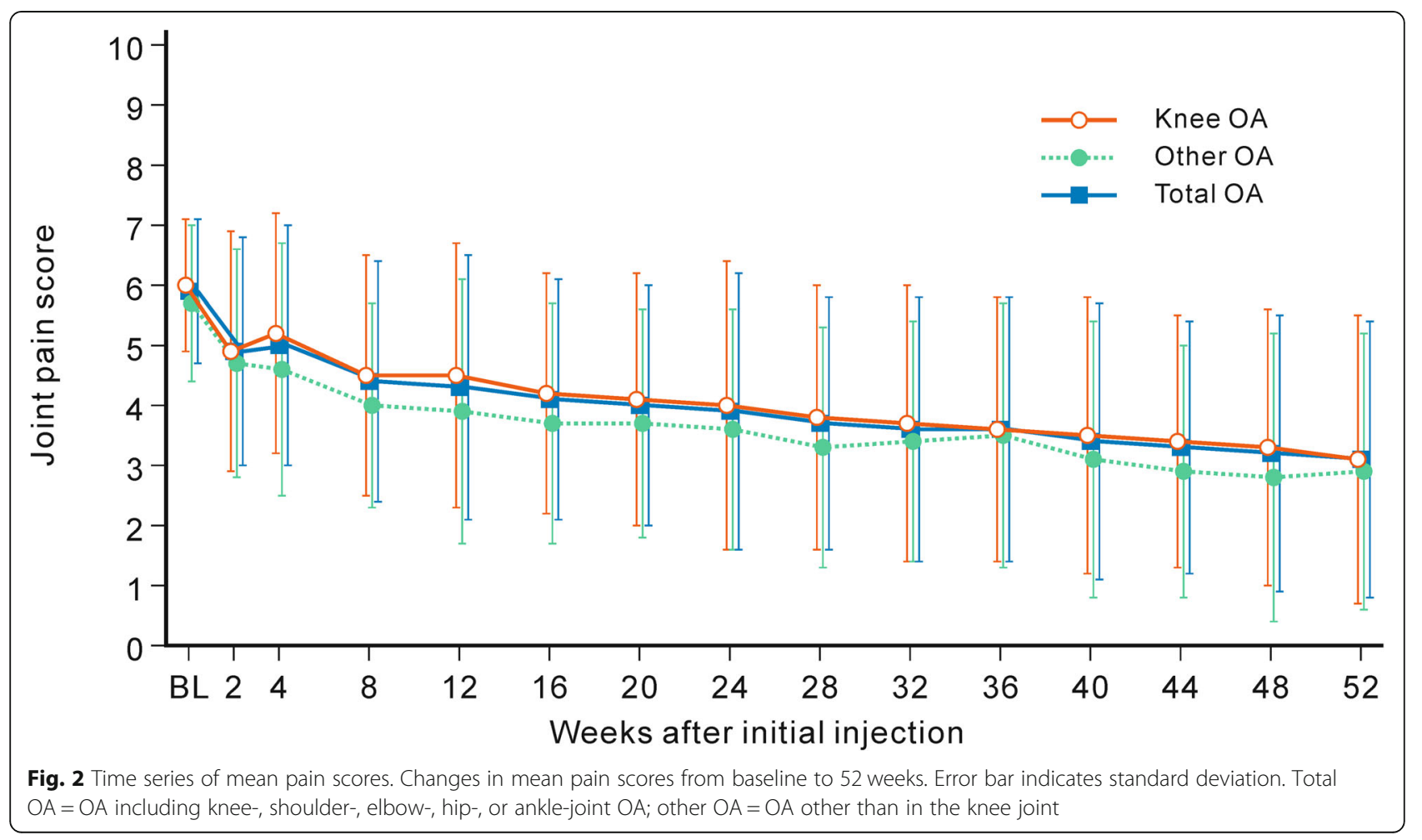


Table 4 Summary statistics of efficacy outcomes at baseline, weeks 2, 12, 24, and 52

\begin{tabular}{|c|c|c|c|c|c|}
\hline & $\begin{array}{l}\text { Baseline } \\
\text { Mean } \pm \text { SD } \\
N=166\end{array}$ & $\begin{array}{l}\text { Week } 2 \\
\text { Mean } \pm \text { SD } \\
N=166\end{array}$ & $\begin{array}{l}\text { Week } 12 \\
\text { Mean } \pm \text { SD } \\
N=162\end{array}$ & $\begin{array}{l}\text { Week } 24 \\
\text { Mean } \pm \text { SD } \\
N=158\end{array}$ & $\begin{array}{l}\text { Week } 52 \\
\text { Mean } \pm \text { SD } \\
N=157\end{array}$ \\
\hline Pain score & $5.9 \pm 1.2$ & $4.9 \pm 1.9$ & $4.3 \pm 2.2$ & $3.9 \pm 2.3$ & $3.1 \pm 2.3$ \\
\hline Patient global assessment (mm) & $54.8 \pm 19.3$ & $43.4 \pm 21.7$ & $36.3 \pm 23.7$ & $33.0 \pm 22.7$ & $24.9 \pm 23.0$ \\
\hline Physician global assessment (mm) & $54.2 \pm 15.5$ & $41.6 \pm 19.8$ & $32.8 \pm 20.7$ & $31.2 \pm 22.1$ & $23.7 \pm 21.6$ \\
\hline \multicolumn{6}{|l|}{$\mathrm{SF}-36^{\mathrm{a}}$} \\
\hline MCS & $53.0 \pm 8.4$ & NA & $53.1 \pm 9.5$ & $53.7 \pm 9.4$ & $52.8 \pm 9.2$ \\
\hline RCS & $47.5 \pm 13.3$ & NA & $49.0 \pm 11.7$ & $49.8 \pm 12.1$ & $47.9 \pm 12.5$ \\
\hline PCS & $31.9 \pm 11.1$ & NA & $33.0 \pm 12.1$ & $33.9 \pm 13.0$ & $36.3 \pm 12.4$ \\
\hline \multicolumn{6}{|l|}{$E Q-5 D^{a}$} \\
\hline QOL score & $0.73 \pm 0.13$ & NA & $0.77 \pm 0.13$ & $0.78 \pm 0.14$ & $0.78 \pm 0.14$ \\
\hline VAS score & $68.4 \pm 15.6$ & NA & $68.8 \pm 18.0$ & $70.9 \pm 18.3$ & $71.1 \pm 18.6$ \\
\hline Responder $^{\mathrm{b}}, \mathrm{n}(\%)$ & NA & $49(29.5)$ & $76(46.9)$ & $91(57.6)$ & $108(68.8)$ \\
\hline
\end{tabular}

SF-36 Medical Outcomes Study Short-Form 36-Item Health Survey, MCS mental component summary, NA not applicable, RCS role/social component summary, PCS physical component summary, EQ-5D EuroQol 5 dimension, $Q O L$ quality of life, VAS visual analogue scale

${ }^{a}$ Lower score indicates more pain or functional disability, and higher score indicates less pain or functional disability. QOL assessment was not conducted at week 2

${ }^{\mathrm{b}}$ Patients with improvement in joint pain score from baseline of at least $30 \%$

and the time course of improvement in WOMAC pain score over 24 weeks in this study was similar to that of the confirmatory phase 3 study (unpublished results). Extrapolating from these similar results, the continuous efficacy of DF-HA might last up to 52 weeks. These safety and efficacy profiles of one injection of DF-HA every 4 weeks indicate the suitability of DF-HA for the treatment of patients with $\mathrm{OA}$, a progressive chronic disease requiring a safe and effective long-term treatment.

Concomitant use of analgesics was prohibited in the phase 2 study [10] and confirmatory phase 3 study but permitted in the current study. Therefore, the safety and efficacy profiles of DF-HA were assessed under such conditions, with consideration of its real-world use.

Table 5 Subgroup analyses in the full analysis set

\begin{tabular}{|c|c|c|c|c|c|}
\hline & $\begin{array}{l}\text { Baseline } \\
\text { Mean } \pm \text { SD }\end{array}$ & $\begin{array}{l}\text { Week } 2 \\
\text { Mean } \pm \text { SD }\end{array}$ & $\begin{array}{l}\text { Week } 12 \\
\text { Mean } \pm \text { SD }\end{array}$ & $\begin{array}{l}\text { Week } 24 \\
\text { Mean } \pm \text { SD }\end{array}$ & $\begin{array}{l}\text { Week } 52 \\
\text { Mean } \pm \text { SD }\end{array}$ \\
\hline \multicolumn{6}{|c|}{ Patients with knee OA or other OA } \\
\hline Knee OA (n) & 126 & 126 & 122 & 121 & 120 \\
\hline Pain score & $6.0 \pm 1.1$ & $4.9 \pm 2.0$ & $4.5 \pm 2.2$ & $4.0 \pm 2.4$ & $3.1 \pm 2.4$ \\
\hline \multicolumn{6}{|c|}{ WOMAC score (mm) } \\
\hline Pain & $40.9 \pm 18.7$ & $33.1 \pm 21.2$ & $29.3 \pm 22.3$ & $26.7 \pm 22.4$ & $21.7 \pm 22.1$ \\
\hline Stiffness & $39.9 \pm 25.9$ & $32.2 \pm 23.6$ & $30.2 \pm 24.5$ & $28.2 \pm 24.7$ & $21.8 \pm 22.6$ \\
\hline Function & $40.5 \pm 21.7$ & $35.0 \pm 23.0$ & $29.4 \pm 22.7$ & $27.2 \pm 23.7$ & $22.0 \pm 22.8$ \\
\hline Total & $40.5 \pm 20.2$ & $34.4 \pm 22.0$ & $29.5 \pm 22.3$ & $27.2 \pm 23.1$ & $21.9 \pm 22.3$ \\
\hline Other OA (n) & 40 & 40 & 40 & 37 & 37 \\
\hline Pain score & $5.7 \pm 1.3$ & $4.7 \pm 1.9$ & $3.9 \pm 2.2$ & $3.6 \pm 2.0$ & $2.9 \pm 2.3$ \\
\hline \multicolumn{6}{|c|}{ Patients with or without analgesics for $O A$} \\
\hline Use $^{a}(n)$ & 135 & 135 & 132 & 129 & 128 \\
\hline Pain score & $5.9 \pm 1.2$ & $4.9 \pm 1.9$ & $4.3 \pm 2.1$ & $4.0 \pm 2.2$ & $3.2 \pm 2.3$ \\
\hline Not used $^{\mathrm{b}}(\mathrm{n})$ & 31 & 31 & 30 & 29 & 29 \\
\hline Pain score & $5.7 \pm 1.2$ & $4.7 \pm 2.2$ & $4.3 \pm 2.4$ & $3.7 \pm 2.5$ & $2.4 \pm 2.4$ \\
\hline
\end{tabular}

$n$ number of patients in the subgroup, OA osteoarthritis, WOMAC Western Ontario and McMaster University Osteoarthritis 3.1 index

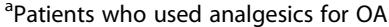

${ }^{\mathrm{b}}$ Patients who did not use analgesics for OA 
Most concomitant analgesics were oral or topical NSAI Ds, which are recommended in the guidelines for OA although oral NSAIDs have a risk of GI disorders [3]. The incidence of TEAEs was higher in patients treated concomitantly with NSAIDs than that in those without NSAIDs. However, some patients used NSAIDs for the management of TEAEs such as infection and pain irrelevant to $\mathrm{OA}$, which was considered the cause of the high incidence of TEAEs in this study. In addition, there was no difference in the incidence of GI disorders reflecting major adverse reactions of NSAIDs between patients treated concomitantly with or without NSAIDs. The low systemic exposure to DF released from DF-HA may have contributed to this result. Regarding efficacy, similar changes in pain scores were observed regardless of the combination of analgesics. This suggests that DF-HA is efficacious in patients receiving NSAIDs with the same mechanism of action but different usage. This might be because DF-HA directly releases DF into the painful site of the joint cavity, in addition to the effect of HA. When oral and topical DF was administered to knee OA patients, the mean concentrations of DF were 15.07 and $4.99 \mathrm{ng} / \mathrm{g}$ in the synovium, and 16.76 and $1.96 \mathrm{ng} / \mathrm{mL}$ in the synovial fluid $12 \mathrm{~h}$ after administration, respectively [24]. One dose of DF-HA (30 mg) contains $3.5 \mathrm{mg}$ of DF [8] and the mean synovial fluid volume is approximately $3 \mathrm{~mL}$ in the human knee [25]; therefore, DF-HA IA injection might achieve a higher local concentration of DF for a longer period in the knee synovial fluid than its oral or topical administration, though the actual time course of DF concentration in the synovium or synovial fluid after DF-HA administration in OA patients has not been measured. The mean joint pain score (NRS score) was $<4$ in patients receiving DF-HA with or without analgesics. An NRS score $<4$ indicates a patient with an acceptable symptom state [26] and a clinically significant improvement from baseline. On the basis of these safety and efficacy results of DF-HA concomitant with NSAI Ds, DF-HA may be used in combination with oral or topical NSAIDs, or as DF-HA monotherapy.

$\mathrm{OA}$ is a degenerative joint disorder that develops in various joints. The treatment options for OA are similar regardless of the affected joint. A typical medical treatment is pain management with analgesics such as NSAI Ds. However, IA injection, which is widely used for knee OA patients, is not a common treatment option for other joints, and data of its efficacy are limited [27-32]. One of the aims of this study was to use DF-HA for various OA; therefore, patients with knee, shoulder, elbow, hip, and ankle OA were enrolled to evaluate the safety and efficacy of DF-HA. Although the interpretation of the results is limited because of the small sample size of the other OA than knee groups, similar trends in safety and efficacy were observed between knee and other joints. Additional trials are needed to determine which joint OA is suitable for DF-HA treatment with an acceptable safety and efficacy profile.

This study reports safety and efficacy data for DF-HA injected repeatedly for 1 year, and provides useful information for its practical use to treat patients with OA joints, and its combined use with NSAIDs and HA. However, this study had several limitations: 1) it was difficult to accurately evaluate the safety and efficacy of DF-HA because this study had no control arm and a large placebo effect was reported for IA injection in OA [33]; 2) no restricted concomitant use of analgesics, including NSAIDs, and IA-HA at the same target joint might have affected the results of the study; 3) sample sizes of subgroups of individuals with joint OA other than knee OA, including patients treated with DF-HA in combination with NSAIDs and/or HA, were too low to evaluate the safety and efficacy or identify risk groups; and regarding IA-HA subgroup analysis, it is also a limitation that the analysis was conducted to evaluate only safety in a small number of patients; 4) treatment/observation periods do not necessarily seem to be enough to evaluate the risk of cartilage loss or OA progression, which is a concern in patients receiving long-term treatment with NSAIDs and IA corticosteroids [20, 21]. Therefore, a well-controlled study conducted over a longer period is required to evaluate the efficacy and safety of DF-HA when employed as a long-term IA-injectable drug. In addition, it will be necessary to evaluate the effect of the difference in patient demographic characteristics, such as K-L grade, on the efficacy and safety of DF-HA to address future clinical questions.

\section{Conclusions}

In the present study, repeated IA injection of DF-HA every 4 weeks for 1 year was tolerated well with no clinically significant adverse events and was suggested to lead to the long-term improvement of OA symptoms. DF-HA may be a useful treatment for patients with OA.

\section{Abbreviations}

BMI: Body mass index; C-IRB: Central Institutional Review Board; DFHA: Diclofenac etalhyaluronate; EQ-5D: EuroQol 5 dimension; FAS: Full analysis set; GI: Gastrointestinal; HA: Hyaluronan; IA: Intra-articular; ICH: International Conference on Harmonization; K-L: Kellgren and Lawrence: MCS: Mental component summary; MedDRA: Medical Dictionary for Regulatory Activities; NA: Not applicable; NRS: Numerical rating scale; NSAI Ds: Nonsteroidal anti-inflammatory drugs; OA: Osteoarthritis; PCS: Physical component summary; PREE-J: Patient-Rated Elbow Evaluation-Japanese Version; QOL: Quality of life; RCS: Role/social component summary; SAFE-Q: SelfAdministered Foot Evaluation Questionnaire; SD: Standard deviation; SF36: Medical Outcomes Study 36-Item Short-Form Health Survey;

TEAEs: Treatment-emergent adverse events; VAS: Visual analogue scale; WOMAC: Western Ontario and McMaster University Osteoarthritis 3.1 index

\section{Acknowledgements}

The authors thank the patients who participated in the clinical trial and all study investigators, study coordinators, and other study personnel who participated in the study. Medical writing and editorial support were 
provided by ASCA Corporation funded by Seikagaku Corporation and Ono Pharmaceutical Co., Ltd.

\section{Authors' contributions}

All authors contributed to the design of the study, interpreted the data, and discussed, reviewed and agreed on the content of the final manuscript before submission. YN provided important clinical input regarding osteoarthritis. KK and TO conducted the study. TS conducted the data analysis

\section{Funding}

This study was sponsored by the Seikagaku Corporation and Ono Pharmaceutical Co., Ltd. Medical writing support and editorial support was funded by Seikagaku Corporation and Ono Pharmaceutical Co., Ltd.

\section{Availability of data and materials}

The datasets generated and analysed during the current study are not publicly available due to confidential nature of the material, but are available from the corresponding author on reasonable request.

\section{Declarations}

\section{Ethics approval and consent to participate}

This study was approved by institutional review boards (Shinagawa East One Medical Clinic IRB, Adachi Kyosai Hospital IRB, Yokohama Minoru Clinic IRB, National Hospital Organization Tokushima Hospital IRB, Sone Clinic IRB) and conducted in accordance with the Declaration of Helsinki and the International Council for Harmonization Good Clinical Practice Guidelines. All patients provided written informed consent before being enrolled in this study.

\section{Consent for publication}

Not applicable.

\section{Competing interests}

YN received consulting fees from Seikagaku Corporation, during the conduct of the study; grants from Daiichi Sankyo Company, Limited, Novartis International AG, Pfizer Inc., Eisai Co., Ltd., Zimmer-Biomet, and Chugai Pharmaceutical Co., Ltd., consulting fees from Eli Lilly Japan K. K, lecture fees from Kaken Pharmaceutical Co. Ltd., Hisamitsu Pharmaceutical Co. Inc., Kyowa Kirin Co., Ltd., and Asahi Kasei Pharma Corporation, outside the submitted work. KK, TO, and TS are employees of Seikagaku Corporation.

\section{Author details}

${ }^{1}$ Department of Rehabilitation, Orthopaedic Surgery, Nagoya University Hospital, 65 Tsurumai-cho, Showa-ku, Nagoya, Aichi 466-8560, Japan. ${ }^{2}$ Clinical Development Department, Research \& Development Division, Seikagaku Corporation, Chiyoda-ku, Tokyo, Japan.

Received: 16 November 2020 Accepted: 22 February 2021 Published online: 01 March 2021

\section{References}

1. Vennu V, Bindawas SM. Relationship between falls, knee osteoarthritis, and health-related quality of life: data from the osteoarthritis initiative study. Clin Interv Aging. 2014;9:793-800.

2. Kolasinski SL, Neogi T, Hochberg MC, Oatis C, Guyatt G, Block J, et al. 2019 American College of Rheumatology/Arthritis Foundation guideline for the management of osteoarthritis of the hand, hip, and knee. Arthritis Care Res (Hoboken). 2020;72:149-62.

3. Bannuru RR, Osani MC, Vaysbrot EE, Arden NK, Bennell K, Bierma-Zeinstra SMA, et al. OARSI guidelines for the non-surgical management of knee, hip, and polyarticular osteoarthritis. Osteoarthr Cartil. 2019;27:1578-89.

4. Crofford LJ. Use of NSAIDs in treating patients with arthritis. Arthritis Res Ther. 2013;15:S2

5. Kompel AJ, Roemer FW, Murakami AM, Diaz LE, Crema MD, Guermazi A. Intra-articular corticosteroid injections in the hip and knee: perhaps not as safe as we thought? Radiology. 2019;293:656-63.

6. Matzkin EG, Curry EJ, Kong Q, Rogers MJ, Henry M, Smith EL. Efficacy and treatment response of intra-articular corticosteroid injections in patients with symptomatic knee osteoarthritis. J Am Acad Orthop Surg. 2017:25:703-14.

7. Deyle GD, Allen CS, Allison SC, Gill NW, Hando BR, Petersen EJ, et al. Physical therapy versus glucocorticoid injection for osteoarthritis of the knee. N Engl J Med. 2020;382:1420-9.

8. Yoshioka K, Kisukeda T, Zuinen R, Yasuda Y, Miyamoto K. Pharmacological effects of N-[2-[[2-[2-[(2,6-dichlorophenyl)amino]phenyl]acetyl]oxy]ethyl] hyaluronamide (diclofenac etalhyaluronate, SI-613), a novel sodium hyaluronate derivative chemically linked with diclofenac. BMC Musculoskelet Disord. 2018:19:157.

9. Kisukeda T, Onaya J, Yoshioka K. Effect of diclofenac etalhyaluronate (SI-613) on the production of high molecular weight sodium hyaluronate in human synoviocytes. BMC Musculoskelet Disord. 2019;20:201.

10. Nishida Y, Kano K, Nobuoka Y, Seo T. Sustained-release diclofenac conjugated to hyaluronate (diclofenac etalhyaluronate) for knee osteoarthritis: a randomized phase 2 study. Rheumatology (Oxford). 2020: keaa605. doi: https://doi.org/10.1093/rheumatology/keaa605.

11. Fukuhara S, Bito S, Green J, Hsiao A, Kurokawa K. Translation, adaptation, and validation of the SF-36 health survey for use in Japan. J Clin Epidemiol. 1998;51:1037-44.

12. Fukuhara S, Ware JE, Kosinski M, Wada S, Gandek B. Psychometric and clinical tests of validity of the Japanese SF-36 health survey. J Clin Epidemiol. 1998;51:1045-53.

13. Fukuhara S, Suzukamo Y. Manual of SF-36v2 Japanese version. Kyoto: Institute for Health Outcomes and Process Evaluation Research; 2004.

14. Herdman M, Gudex C, Lloyd A, Janssen MF, Kind P, Parkin D, et al. Development and preliminary testing of the new five-level version of EQ-5D (EQ-5D-5L). Qual Life Res. 2011:20:1727-36.

15. Bellamy N, Buchanan WW, Goldsmith $\mathrm{CH}$, Campbell J, Stitt LW Validation study of WOMAC: a health status instrument for measuring clinically important patient relevant outcomes to antirheumatic drug therapy in patients with osteoarthritis of the hip or knee. J Rheumatol. 1988;15:1833-40.

16. McAlindon TE, Driban JB, Henrotin Y, Hunter DJ, Jiang GL, Skou ST, et al. OARSI clinical trials recommendations: design, conduct, and reporting of clinical trials for knee osteoarthritis. Osteoarthr Cartil. 2015;23:747-60.

17. Shimo S, Sakamoto Y, Amari T, Gemma S. Validation of the shoulder36 for the activities of daily living with shoulder disorders. J Phys Ther Sci. 2017;29: $635-40$

18. Hanyu T, Watanabe M, Masatomi T, Nishida K, Nakagawa T, Nishiura Y, et al. Reliability, validity, and responsiveness of the Japanese version of the patient-rated elbow evaluation. J Orthop Sci. 2013;18:712-9.

19. Niki H, Tatsunami S, Haraguchi N, Aoki T, Okuda R, Suda Y, et al. Validity and reliability of a self-administered foot evaluation questionnaire (SAFE-Q). J Orthop Sci. 2013:18:298-320.

20. Brandt KD. The role of analgesics in the management of osteoarthritis pain. Am J Ther. 2000:7:75-90.

21. McAlindon TE, LaValley MP, Harvey WF, Price LL, Driban JB, Zhang M, et al. Effect of intra-articular triamcinolone vs saline on knee cartilage volume and pain in patients with knee osteoarthritis: a randomized clinical trial. JAMA. 2017:317:1967-75.

22. Hochberg MC. Serious joint-related adverse events in randomized controlled trials of anti-nerve growth factor monoclonal antibodies. Osteoarthr Cartil. 2015;23(Suppl 1):18-21.

23. Honvo G, Reginster JY, Rannou F, Rygaert X, Geerinck A, Rabenda V, et al. Safety of intra-articular hyaluronic acid injections in osteoarthritis: outcomes of a systematic review and meta-analysis. Drugs Aging. 2019;36(Suppl 1): $101-27$.

24. Miyatake $\mathrm{S}$, Ichiyama H, Kondo E, Yasuda K. Randomized clinical comparisons of diclofenac concentration in the soft tissues and blood plasma between topical and oral applications. Br J Clin Pharmacol. 2009;67: $125-9$.

25. Kraus VB, Stabler TV, Kong SY, Varju G, McDaniel G. Measurement of synovia fluid volume using urea. Osteoarthr Cartil. 2007:15:1217-20.

26. Migliore A, Gigliucci G, Alekseeva L, Avasthi S, Bannuru RR, Chevalier X, et al. Treat-to-target strategy for knee osteoarthritis. International technical expert panel consensus and good clinical practice statements. Ther Adv Musculoskelet Dis. 2019:11:1759720X19893800.

27. Poonit K, Zhou X, Zhao B, Sun C, Yao C, Zhang F, et al. Treatment of osteoarthritis of the elbow with open or arthroscopic debridement: a narrative review. BMC Musculoskelet Disord. 2018;19:394. 
28. McCabe PS, Maricar N, Parkes MJ, Felson DT, O'Neill TW. The efficacy of intra-articular steroids in hip osteoarthritis: a systematic review. Osteoarthr Cartil. 2016;24:1509-17.

29. Burbank KM, Stevenson JH, Czarnecki GR, Dorfman J. Chronic shoulder pain: part II. Treatment Am Fam Physician. 2008;77:493-7.

30. Khlopas H, Khlopas A, Samuel LT, Ohliger E, Sultan AA, Chughtai M, et al. Current concepts in osteoarthritis of the ankle: review. Surg Technol Int. 2019;35:280-94.

31. Zhang B, Thayaparan A, Horner N, Bedi A, Alolabi B, Khan M. Outcomes of hyaluronic acid injections for glenohumeral osteoarthritis: a systematic review and meta-analysis. J Shoulder Elb Surg. 2019;28:596-606.

32. Paik J, Duggan ST, Keam SJ. Triamcinolone acetonide extended-release: a review in osteoarthritis pain of the knee. Drugs. 2019;79:455-62.

33. Zhang W. The powerful placebo effect in osteoarthritis. Clin Exp Rheumatol. 2019;37(Suppl 1):118-23.

\section{Publisher's Note}

Springer Nature remains neutral with regard to jurisdictional claims in published maps and institutional affiliations.

Ready to submit your research? Choose BMC and benefit from:

- fast, convenient online submission

- thorough peer review by experienced researchers in your field

- rapid publication on acceptance

- support for research data, including large and complex data types

- gold Open Access which fosters wider collaboration and increased citations

- maximum visibility for your research: over $100 \mathrm{M}$ website views per year

At BMC, research is always in progress.

Learn more biomedcentral.com/submissions 